Pacific Journal of Mathematics

SPECIAL GENERATING SETS OF PURELY INSEPARABLE
EXTENSION FIELDS OF UNBOUNDED EXPONENT 


\title{
SPECIAL GENERATING SETS OF PURELY INSEPARABLE EXTENSION FIELDS OF UNBOUNDED EXPONENT
}

\author{
B. I. EKE
}

The present paper considers the problem of choosing a maximum subfield having a subbasis over $K$ among subextensions of $L / K$, when $L / K$ is purely inseparable but of unbounded exponent.

Throughout $L$ will be a purely inseparable extension field of a field $K$ of characteristic $p \neq 0$. For the case when $L / K$ is of bounded exponent $e>0$ Weisfeld [6, Theorem 3, p. 442] has shown that among the subfields of $L$ having a subbasis over $K$ there is a maximal subfield with respect to set inclusion. This theorem fails in the unbounded exponent case since such a maximal subfield would not always exist [6, p. 442]. An open problem was, therefore, posed in Weisfeld's paper regarding a necessary and sufficient condition for the theorem to hold for extensions $L / K$ of unbounded exponent. The present paper seeks to provide a solution to this problem.

Let $M$ be a given subset of $L$. The subset $M$ will be said to be in canonical form when $M$ is put in the form $M=A_{1} \cup A_{2} \cup \cdots$ where $A_{i}$ consists of the elements of $M$ having exponent $i$ over $K$. $M$ is called a canonical generating set over $K$ if $M$ is a minimal generating set for $K(M)$ and when $M=A_{1} \cup A_{2} \cup \cdots$ in canonical form, then the subsets $M_{i}$ defined by $M_{i}=\bigcup_{J=i+1}^{\infty} A_{j}, i=0,1, \ldots, M_{0}=M$, satisfy $M_{i}^{p^{i}}$ is a minimal generating set for $K\left(M^{p^{t}}\right) / K$. The set $M$ is called a distinguished subset of $L / K$ if $M$ is a canonical generating set over $K$ and, for each nonnegative integer $n, K \cap L^{p^{n}} \subseteq K^{p}\left(A_{n}^{p} \cup A_{n+1}^{p} \cup \cdots\right)$ where $M=A_{1} \cup A_{2} \cup \cdots$ in canonical form. Finally, $M$ is called a subbasis over $K$ if for every finite subset $\left\{a_{1}, \ldots, a_{r}\right\}$ of $M, K\left(a_{1}, \ldots, a_{r}\right)$ is the tensor product of the simple extensions $K\left(a_{i}\right), i=1, \ldots, r$, and when this happens, the extension $K(M)$ is called an extension having a subbasis over $K$.

The main result is that if $L / K$ is any purely inseparable extension, then $L / K$ has a maximal subfield $J$ having a subbasis over $K$ if and only if $L / K$ has a distinguished subset $M$.

LEMma 1. If $L / K$ has a subbasis, then every subbasis for $L / K$ is distinguished. 
Proof. Let $L / K$ have a subbasis $B=B_{1} \cup B_{2} \cup \cdots$ in canonical form. Let $u$ be any element of $L$ with exponent $n$ over $K$. Then $u^{p^{n-1}} \in K\left(B^{p^{n-1}}\right)=K\left(\bigcup_{i=n}^{\infty} B_{i}^{p^{n-1}}\right)$ which shows that the exponent of $u$ over $K\left(\cup_{i=n}^{\infty} B_{i}\right)$ is less than $n$. Hence $B$ is distinguished.

LEMMA 2. If the subset $M$ of $L$ is a canonical generating set over $K$, then $M$ is a subbasis over $K$.

Proof. Suppose $M$ is a canonical generating set over $K$ but $M$ is not a subbasis over $K$. Let $M=A_{1} \cup A_{2} \cup \cdots$ in canonical form and let $e$ be the smallest positive integer such that there exists an element $b \in A_{e}$ for which $b^{p^{e-1}} \in K(M-b)$. Clearly $e \neq 1$ otherwise we contradict the minimality of $M$ over $K$. There exists a smallest positive integer $t$ such that

$$
b^{p^{e-1}} \in K\left(M_{t-1}-b\right)
$$

where $M_{t-1}=\bigcup_{j=t}^{\infty} A_{j}$. Also there exists an element $a \in A_{t}$ such that $b^{p^{e-1}} \in K\left(M_{t-1}-b\right)$ but

$$
b^{p^{e-1}} \notin K\left(M_{t-1}-\{a, b\}\right) .
$$

Let $s$ be the highest integer such that

$$
b^{p^{e-1}} \in K\left(M_{t-1}-\{a, b\}, a^{p^{s}}\right) .
$$

Then $a^{p^{s}} \in K\left(M_{t-1}-\{a, b\}, a^{p^{s+1}}, b^{p^{e-1}}\right)$. Consequently $a^{p^{s}}$ is separable and purely inseparable over $K\left(M_{t-1}-\{a, b\}, b^{p^{e-1}}\right)$ which says that

$$
a^{p^{s}} \in K\left(M_{t-1}-\{a, b\}, b^{p^{e-1}}\right) .
$$

In expression (1) above it must be the case that $e>t$ and in (4) it is the case that $s \geq t$ both because of [3, Cor. 1.31, p. 28]. But if $s>t$, then in expression (3) we have $K\left(M_{t-1}-\{a, b\}, a^{p^{s}}\right)=K\left(M_{t-1}-\{a, b\}\right)$ so that $b^{p^{e-1}} \in K\left(M_{t-1}-\{a, b\}\right)$ contradicting the expression (2). Therefore $s=t$. So, we have $s=t<e$. But then (4) implies that $a^{p^{t}} \in$ $K\left(M_{t-1}-a\right) \subseteq K(M-a)$ where $t<e$ contradicting the minimality of $e$ for this purpose. This contradiction proves the assertion.

THEOREM 3 (Main result). The extension $L / K$ has a maximal subfield $J$ having a subbasis over $K$ if and only if $L / K$ has a distinguished subset $M$.

Proof. Suppose $L / K$ has a distinguished subset $M$. By Lemma $2 M$ is a subbasis over $K$. Moreover, $M$ is distinguished in $L / K$ implies that any element of $L$ having exponent $r$ over $K$ must have exponent less than $r$ over $K\left(\bigcup_{i=r}^{\infty} A_{i}\right)$ where $M=A_{1} \cup A_{2} \cup \cdots$ in canonical form. 
Denote $K(M)$ by $J$. Let $F$ be any modular subfield of $L$ over $K$ containing $J$ and suppose $u \in F-J$ has exponent $r$ over $k$. Then one can write

$$
u^{p^{s}}=a_{1} u_{1}^{p^{s}}+\cdots+a_{n} u_{n}^{p^{s}}
$$

where $a_{1}, \ldots, a_{n} \in K, u_{1}, \ldots, u_{n} \in J, s<r$, and $n$ is chosen minimal. Using arguments similar to those of Weisfeld in [6, Theorem 4, p. 442] and the concept of $p$-freedom as defined in that paper one can get a maximal $p$-free subset $\left\{a_{1}, \ldots, a_{k}\right\}$ of $\left\{a_{1}, \ldots, a_{n}\right\}$ relative to $J^{p}$ and a maximal $p$-free subset $\left\{a_{1}, \ldots, a_{j}\right\}$ of $\left\{a_{1}, \ldots, a_{k}\right\}$ relative to $F^{p}$ where $j<k$. Consequently we have a relation

$$
\begin{aligned}
& a_{j+1}=\sum\left\{y_{i_{1} \cdots i_{j}}^{p} a_{1}^{i_{1}} \cdots a_{j}^{i_{j}} \mid y_{i_{1} \cdots i_{j}} \in F,\right. \\
& \left.\quad 0 \leq i_{m}<p, m=1, \ldots, j\right\} .
\end{aligned}
$$

Let $B$ be the set consisting of the coefficients $y_{i_{1} \ldots i}$. Let $F_{1}$ be the modular closure of $K(B)$ as defined in [4, p. 408], and let $F_{2}=F \cap F_{1}$. Then $F_{2}$ must have a subbasis over $K$. Therefore by [4, Theorem 1, p. 403] there exists a higher derivation $D$ of $F_{2}$ relative to which $K$ is the field of constants. Using this in (6), one can violate the $p$-freedom of $\left\{a_{1}, \ldots, a_{j}\right\}$ relative to $F^{p}$. Therefore $J=F$.

Conversely let $N$ be a maximal subfield of $L / K$ having a subbasis over $K$ and let $M=A_{1} \cup A_{2} \cup \cdots$ (in canonical form) be any subbasis for $N / K$. As usual, for $i=0,1, \ldots$ we let $M_{i}=\bigcup_{j=i+1}^{\infty} A_{j}$. We must show that $M$ is a distinguished subset of $L / K$. Clearly $M$ is a canonical generating set over $K$. We shall prove, by induction, the statement $P(n)$ : If $u$ is any element of $L$ having exponent $n$ over $K$, then the exponent of $u$ over $K\left(M_{n-1}\right)$ is less than $n$. Now $P(1)$ is trivial. Hence assume $P(n-1)$ holds and suppose an element $u \in L$ has exponent $n$ over $K$ and same exponent over $K\left(M_{n-1}\right)$. Let $A=\{u\} \cup M_{n-1}$. Then $A$ is a subbasis over $K$. Let $T^{(n-1)}=\left\{B \subseteq A_{n-1} \cup A \mid B \supseteq A\right.$ and $B$ is a subbasis over $K$ \}. Clearly $A$ is in $T^{(n-1)}$. So, $T^{(n-1)} \neq \varnothing$. Let $M^{(n-1)}$ be a maximal element (with respect to set inclusion) of the set $T^{(n-1)}$. We now proceed to let $M^{(n-2)}$ be a maximal element of $T^{(n-2)}=\left\{B \subseteq A_{n-2} \cup M^{(n-1)} \mid B \supseteq M^{(n-1)}\right.$ and $B$ is a subbasis over $\left.K\right\}$.

In general, for $1 \leq k<n-1$, we let $M^{(k)}$ be a maximal element of

$$
T^{(k)}=\left\{B \subseteq A_{k} \cup M^{(k+1)} \mid B \supseteq M^{(k+1)} \text { and } B \text { is a subbasis over } K\right\} .
$$

It is our ambition to show that $K\left(M^{(1)}\right)=N$. 
Let $v$ be an element of $M$ and suppose $v \in A_{r}(1 \leq r<n)$. If $v \notin K\left(M^{(r)}\right)$, then it must be the case that $v$ has an exponent $s<r$ over $K\left(M^{(r)}\right)$ by the definition of $M^{(r)}$. Consequently we can write

$$
v^{p^{s}}=c_{1} v_{1}^{p^{s}}+\cdots+c_{m} v_{m}^{p^{s}}
$$

where $c_{1}, \ldots, c_{m} \in K, v_{1}, \ldots, v_{m} \in K\left(M^{(r)}\right), s<r$, and $m$ is minimal. This relation now allows us to apply an argument similar to that in the first part of this proof between $K\left(M^{(r)}\right)$ as $J$ and the modular closure of $K\left(M^{(r)}, v\right)$ as $F$ ( $F$ and $J$ in this case both contained in their composite $F(J)$ as $L)$. The contradiction which will then arise as in the first part shows that $v \in K\left(M^{(r)}\right)$. Consequently, $K\left(M^{(1)}\right)$ contains $K(M)=N$, and, by the maximality of $N, K\left(M^{(1)}\right)=N$. This shows that $u \in N$. By Lemma 1 the exponent of $u$ over $K\left(M_{n-1}\right)$ is less than $n$. This shows that $M$ is a distinguished subset of $L / K$.

COROLlary 4. Let $J$ be a subfield of $L / K$ having a subbasis over $K$. Then $J$ is a maximal subfield of $L / K$ having a subbasis over $K$ if and only if $J \cap K^{p^{-t}}$ is a maximal subfield of $L \cap K^{p^{-i}}$ having a subbasis over $K$, $i=1, \ldots$.

Proof. Let $J$ be a maximal subfield of $L / K$ having a subbasis over $K$, and let $B=B_{1} \cup B_{2} \cup \cdots$ (in canonical form) be a subbasis for $J / K$. Fix the integer $i \geq 1$ and let $B_{(l)}=\left\{a^{p^{s-i}} \mid a \in B_{s}\right.$ and $\left.s>i\right\}$. Then $W=B_{1} \cup \cdots \cup B_{l} \cup B_{(l)}$ is a subset of $J \cap K^{p^{-t}}$ which is also a subbasis over $K$. We shall show that $W$ is a distinguished subset of $L \cap K^{p^{-1}} / K$. Let $u \in L \cap K^{p^{-t}}$ have exponent $e \leq i$ over $K$. We note that by Theorem 3 the subbasis $B$ is a distinguished subset of $L / K$. Let

$$
u^{p^{e-1}}=\sum c_{l_{1} \cdots i_{n}} u_{1}^{i_{1}} \cdots u_{n}^{i_{n}}
$$

where $0 \leq i_{k}<p^{e_{k}}, e_{k}=$ exponent of $u_{k}$ over $K$, and $u_{k} \in \bigcup_{j=e}^{\infty} B_{J}$. Since $u^{p^{e}} \in K$ it must be the case that $i_{k} \geq p^{e_{k}-1}$, and since $e_{k}-i \leq$ $e_{k}-1$, it must be the case that $p^{e_{k}-i} \leq p^{e_{k}-1} \leq i_{k}<p^{e_{k}}$ whenever $e_{k}>$ $i$. Hence $u_{k}^{i_{k}} \in K\left(B_{(i)}\right)$ when $e_{k}>i$ and, of course, $u_{k} \in B_{e_{k}}$ if $e_{k} \leq i$. This shows that if $W=\tilde{B}_{1} \cup \cdots \cup \tilde{B}_{i}$ in canonical form, then $u^{p^{e-1}} \in$ $K\left(\tilde{B}_{e} \cup \cdots \cup \tilde{B}_{i}\right)$. Consequently $W$ is distinguished in $L \cap K^{p^{-1}} / K$ and, by Theorem 3, K(W) is a maximal subfield of $L \cap K^{p^{-1}}$ having a subbasis over $K$. Now it is obvious that $K(W) \subseteq J \cap K^{p^{-t}}$. Now let $x \in$ $J \cap K^{p^{-t}}$. Then $x=\sum a_{i_{1} \cdots i_{m}} v_{1}^{l_{1}} \cdots v_{m}^{l_{m}}$ where $0 \leq l_{J}<p^{e_{j}}, e_{J}=$ exponent of $v_{j}$ over $K$, and $v_{j} \in B, 1 \leq j \leq m$. Since $x^{p^{t}} \in K$ we must have, for each $j, l_{j} \geq p^{e_{-}-i}$ and hence $v_{j}^{l_{j}} \in K(W)$. This shows $J \cap K^{p^{-l}} \subseteq$ $K(W)$, and equality follows. 
Conversely suppose $J \cap K^{p^{-1}}$ is a maximal subfield of $L \cap K^{p^{-i}}$ having a subbasis over $K$. Let $T$ be any subfield of $L / K$ having a subbasis over $K$ and suppose $T \supseteq J$. Then for each $i T \cap K^{p^{-i}} \supseteq J \cap K^{p^{-i}}$. If $T \cap K^{p^{-i}} \neq J \cap K^{p^{-i}}$ we contradict the maximality of $J \cap K^{p^{-i}}$ as stated earlier since $T \cap K^{P^{-1}}$ is also a subfield of $L \cap K^{p^{-i}}$ having a subbasis over $K$. Consequently $J=T$.

It was shown in Lemma 1 that if $L / K$ has a subbasis over $K$, then that subbasis must be a distinguished subset of $L / K$. It is not true, however, that an extension $L / K$ must be modular in order to have a distinguished subset as the following example shows.

EXAMPLE. Let $K=Z_{p}\left(x_{1}, x_{2}, \ldots\right)$ where the $x_{i}$ are algebraically independent indeterminates over $Z_{p}$. Let

$$
L=K\left(x_{1}^{p^{-1}} x_{3}^{p^{-2}}+x_{2}^{p^{-1}}, x_{3}^{p^{-2}}, x_{3}^{p^{-3}}, x_{4}^{p^{-3}}, x_{5}^{p^{-4}}, \ldots\right) .
$$

First, we show that $L / K$ is not modular. We note that

$$
\begin{gathered}
L^{p}=K^{p}\left(x_{1} x_{3}^{p-1}+x_{2}, x_{3}^{p^{-1}}, x_{4}^{p^{-2}}, x_{5}^{p^{-3}}, \ldots\right) \\
=Z_{p}\left(x_{1}^{p}, x_{2}^{p}, x_{1} x_{3}^{p^{-1}}+x_{2}, x_{3}^{p^{-1}}, x_{4}^{p^{-2}}, x_{5}^{p^{-3}}, \ldots\right) . \\
K \cap L^{p}=Z_{p}\left(x_{1}^{p}, x_{2}^{p}, x_{3}, x_{4}, \ldots\right)=K^{p}\left(x_{3}, x_{4}, x_{5}, \ldots\right) .
\end{gathered}
$$

Now the set $\left\{1, x_{3}^{p^{-1}}, x_{1} x_{3}^{p^{-1}}+x_{2}\right\}$ is a subset of $L^{p}$ which is linearly independent over $K \cap L^{p}$. For suppose $c_{0}+c_{1} x_{3}^{p^{-1}}+c_{2} x_{1} x_{3}^{p^{-1}}+c_{2} x_{2}=$ $0, c_{i} \in K \cap L^{p}$ and not both $c_{1}$ and $c_{2}$ are zero. We have

$$
c_{0}^{p}+c_{1}^{p} x_{3}+c_{2}^{p} x_{1}^{p} x_{3}+c_{2}^{p} x_{2}^{p}=0
$$

or

$$
\left(c_{1}^{p}+c_{2}^{p} x_{1}^{p}\right) x_{3}=-\left(c_{2}^{p} x_{2}^{p}+c_{0}^{p}\right) .
$$

If $c_{1}^{p}+c_{2}^{p} x_{1}^{p} \neq 0$, then

$$
x_{3}=\frac{-\left(c_{2}^{p} x_{2}^{p}+c_{0}^{p}\right)}{c_{1}^{p}+c_{2}^{p} x_{1}^{p}} \in K^{p}=Z_{p}\left(x_{1}^{p}, x_{2}^{p}, x_{3}^{p}, \ldots\right) .
$$

There exists a finite $n$ such that

$$
x_{3} \in Z_{p}\left(x_{1}^{p}, \ldots, x_{n}^{p}\right) \subseteq Z_{p}\left(x_{1}, x_{2}, x_{3}^{p}, x_{4}, \ldots, x_{n}\right) .
$$

Consequently $x_{3}$ is separable algebraic over $Z_{p}\left(x_{1}, x_{2}, x_{4}, \ldots, x_{n}\right)$ violating the algebraic independence of the $x_{i}$ over $Z_{p}$. Therefore $c_{1}^{p}+c_{2}^{p} x_{1}^{p}=0$. This again leads to a contradiction unless $c_{1}=c_{2}=0$. Consequently we must have $c_{0}=c_{1}=c_{2}=0$ as required. On the other hand, it is obvious 
that the given set $\left\{1, x_{3}^{p^{-1}}, x_{1} x_{3}^{p^{-1}}+x_{2}\right\}$ is linearly dependent over $K$. This shows that $L / K$ is not modular.

Now the set $S=\left\{x_{3}^{p^{-2}}, x_{4}^{p^{-3}}, x_{5}^{p^{-4}}, \ldots\right\}$ is a subbasis over $K$. Besides, $S$ is distinguished in $L / K$.

Definition. An extension field $F / K$ is called Galois if it is modular and $\bigcap_{i=1}^{\infty} K\left(F^{p^{i}}\right)=K$.

LEMMA 5. If a purely inseparable extension $F / K$ has a subbasis then it is Calois.

Proof. Let $M=B_{1} \cup B_{2} \cup \ldots$ (in canonical form) be a subbasis for $F / K$. Let $x \in \bigcap_{i=1}^{\infty} K\left(F^{p^{i}}\right)$. Then $x=g\left(b_{1}^{p^{1}}, \ldots, b_{n}^{p^{t}}\right)$ for some $b_{1}, \ldots, b_{n}$ $\in M_{i}=\bigcup_{j=i+1}^{\infty} B_{j}$ and $n$ is chosen minimum. Since $M=\bigcup_{j=1}^{\infty} B_{j}$ is part of a linear basis for $F / K$ the set $\left\{b_{1}, \ldots, b_{n}\right\}$ must be contained in every $M_{i}$ otherwise we contradict the unique representation of $x$ relative to the said linear basis. This shows that

$$
x \in K\left(\bigcap_{i=1}^{\infty} F^{p^{i}}\right)=K\left(\bigcap_{i=1}^{\infty} M_{i}^{p^{i}}\right)=K
$$

since $\bigcap_{i=1}^{\infty} M_{i}=\varnothing$. This shows $\bigcap_{i=1}^{\infty} K\left(F^{p^{i}}\right)=K$ and $F / K$ is Galois.

THEOREM 6. The purely inseparable extension $L / K$ has a maximal subfield $F$ having a subbasis over $K$, if and only if there exist in $L$ a maximal modular subfield $F$ which is Galois over $K$.

Proof. Suppose $F$ is a maximal modular subfield of $L / K$ which is also Galois over $K$. Let $A_{1}, A_{2}, \ldots$ be subsets of $F$ constructed in the manner of [2, Theorem 11, p. 339]. Let

$$
Q=\bigcap_{i=1}^{\infty} K\left(F^{p^{i}}\right) \otimes K\left(A_{1} \cup A_{2} \cup \cdots\right)
$$

as defined in [2, Theorem 13]. Then $F$ is relatively perfect over $Q$ and has a subbasis over $Q$. By Lemma $5, F=\bigcap_{i=1}^{\infty} Q\left(F^{p^{i}}\right)=Q$. From the fact that $F / K$ is also Galois we have

$$
F=Q=\bigcap_{i=1}^{\infty} K\left(F^{p^{i}}\right) \otimes K\left(A_{1} \cup A_{2} \cup \cdots\right)=K\left(A_{1} \cup A_{2} \cup \cdots\right) .
$$

Consequently $F$ has a subbasis over $K$. The converse is immediate. 


\section{REFERENCES}

[1] G. F. Haddix, J. N. Mordeson, and B. Vinograde, On purely inseparable extensions of unbounded exponent, Canad. J. Math., XXI (1969), 1526-1532.

[2] L. A. Kime, Purely inseparable, modular extensions of unbounded exponent, Trans. Amer. Math. Soc., 176 (1973), 335-349.

[3] J. N. Mordeson and B. Vinograde, Structure of Arbitrary Purely Inseparable Extension Fields, Lecture Notes in Math. Vol. 173, Springer-Verlag, New York (1970).

[4] M. Sweedler, Structure of inseparable extensions, Ann. of Math., 87 (1968), 401-410.

[5] Correction to "Structure of inseparable extensions", Ann. of Math., 89 (1969), 206-207.

[6] M. Weisfeld, Purely inseparable extensions and higher derivations, Trans. Amer. Math. Soc., 116 (1965), 435-449.

Received January 24, 1985.

INSTITUE OF MANAGEMENT AND TECHNOLOGY

ENUGU, NigERIA 



\section{PACIFIC JOURNAL OF MATHEMATICS EDITORS}

\author{
V. S. VARADARAJAN \\ (Managing Editor) \\ University of California \\ Los Angeles, CA 90024 \\ HERBERT Clemens \\ University of Utah \\ Salt Lake City, UT 84112 \\ R. FINN \\ Stanford University \\ Stanford, CA 94305
}

HERMANN FLASCHKA

University of Arizona

Tucson, AZ 85721

RAMESH A. GANGOLLI

University of Washington

Seattle, WA 98195

VAughan F. R. JONES

University of California

Berkeley, CA 94720

ROBION KIRBY

University of California

Berkeley, CA 94720
C. C. MoOrE

University of California

Berkeley, CA 94720

H. SAMELSON

Stanford University

Stanford, CA 94305

HAROLD STARK

University of California, San Diego

La Jolla, CA 92093

\section{ASSOCIATE EDITORS}
R. AREnS
E. F. BECKENBACH
B. H. NEUMANN
F. WOLF
K. YOSHIDA (1906-1982)

\section{SUPPORTING INSTITUTIONS}

UNIVERSITY OF ARIZONA

UNIVERSITY OF BRITISH COLUMBIA

CALIFORNIA INSTITUTE OF TECHNOLOGY

UNIVERSITY OF CALIFORNIA

MONTANA STATE UNIVERSITY

UNIVERSITY OF NEVADA, RENO

NEW MEXICO STATE UNIVERSITY

OREGON STATE UNIVERSITY
UNIVERSITY OF OREGON UNIVERSITY OF SOUTHERN CALIFORNIA STANFORD UNIVERSITY UNIVERSITY OF HAWAII UNIVERSITY OF TOKYO UNIVERSITY OF UTAH WASHINGTON STATE UNIVERSITY UNIVERSITY OF WASHINGTON 


\section{Pacific Journal of Mathematics}

\section{Vol. 128, No. $1 \quad$ March, 1987}

Anthony Peter Bahri and Peter Gilkey, The eta invariant, $\operatorname{Pin}^{c}$ bordism, and equivariant $\operatorname{Spin}^{c}$ bordism for cyclic 2-groups $\ldots \ldots \ldots \ldots \ldots \ldots 1$

Friedrich-Wilhelm Bauer, Extensions of generalized homology theories . . . 25

Marilyn Breen, A characterization theorem for compact unions of two

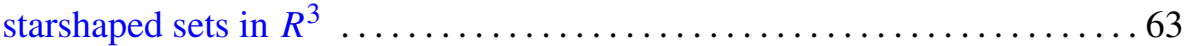

Boniface Ihemotuonye Eke, Special generating sets of purely inseparable extension fields of unbounded exponent $\ldots \ldots \ldots \ldots \ldots \ldots \ldots \ldots \ldots \ldots$

Robert William Gilmer, Jr. and William James Heinzer, Jónsson $\omega_{0}$-generated algebraic field extensions $\ldots \ldots \ldots \ldots \ldots \ldots \ldots \ldots \ldots . . .61$

Guido Lupacciolu, Holomorphic continuation in several complex variables

Douglas C. McMahon, Jaap C. S. P. van der Woude and Ta-Sun Wu,

Connectedness related to almost periodicity of compositions of flow

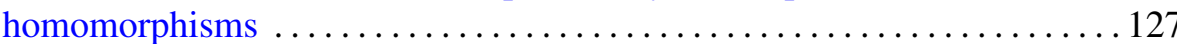

Sophocles Mercourakis, Some characterizations of analytic metric spaces

Peter Frederick Stiller, The Picard numbers of elliptic surfaces with many

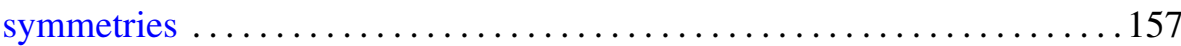

David J. Winter, Reducible complements of Lie algebra radicals ........ 191 Rade Živaljević, On a cohomology theory based on hyperfinite sums of microsimplexes 